\title{
Mosaic genome-wide paternal uniparental disomy
}

INSERM

\section{Source}

INSERM. (1999). Orphanet: an online rare disease and orphan drug data base. Mosaic genome-wide paternal uniparental disomy. ORPHA:329813

A rare chromosomal anomaly characterized by a combination of paternal uniparental and biparental cell lineages, leading to variable clinical presentation that predominantly includes features of Beckwith-Wiedemann syndrome and increased risk of various tumors. In addition, features of Angelman syndrome and transient neonatal diabetes might be expected. 